\title{
A homogeneous HLA-B*27 genotyping assay using dried reagent mixtures
}

\author{
Minna Kiviniemi ${ }^{\mathrm{a}, *}$, Jorma Ilonen $^{\mathrm{a}, \mathrm{b}}$ and Timo Lövgren ${ }^{\mathrm{c}}$ \\ ${ }^{a}$ Immunogenetics Laboratory, University of Turku, Tykistökatu 6 A, 4th floor, FIN-20520 Turku, Finland \\ ${ }^{\mathrm{b}}$ Department of Clinical Microbiology, University of Kuopio and Biocenter Kuopio, FIN-70211, Kuopio, Finland \\ ${ }^{\mathrm{c}}$ Department of Biotechnology, University of Turku, Tykistökatu 6 A, 6th floor, FIN-20520 Turku, Finland
}

\begin{abstract}
The presence of HLA-B*27 allele with patients suspected with ankylosing spondylitis can be used in the diagnostic process. We have developed an assay for typing for the HLA-B*27 in whole blood dried on sample collection cards using pre-dried reagent wells and homogeneous time-resolved fluorescence based PCR approach. Essentially only the sample needs to be added to the dry ready-to-use reaction well in order to start the homogenous amplification assay. The method was validated with 229 samples also typed with an existing DELFIA-based method and results of both assays were 100\% concordant. The dried reagents were shown to be stable at least up to eight weeks at room temperature without any decline in their performance.
\end{abstract}

Keywords: Dry chemistry, HLA-B*27, homogeneous assay, PCR

\section{Introduction}

The major application of genotyping for human leukocyte antigens (HLA) is identification of compatible donor recipient pairs for tissue transplantation. Demanding high resolution full-house approaches, with as detailed genotyping of an individual as possible, are commonly used to ensure as complete identity as possible. Besides tissue transplantation definition of certain HLA alleles and allele combinations is used for diagnostic purposes making use of the known disease associations. Typing of only one or few alleles possibly at low level of resolution allows application of methodologies more suitable for handling of large sample numbers and automation.

The association of HLA-B*27 with ankylosing spondylitis (AS) is one of the strongest associations and the allele is also found increased among several types of postinfectious reactive arthritides; around $90 \%$ of AS patients carry this allele compared to approximately $9 \%$ of controls $[1,3,11,17]$. Typing for the presence

*Corresponding author: M. Kiviniemi. Tel.: +358 2333 7009; Fax: +358 2 3337000; E-mail: minna.kiviniemi@utu.fi. of HLA-B*27 is thus widely used in diagnostic laboratories using a multitude of methods including serological and different sequence based techniques such as PCR with sequence-specific primers [14], PCR-RFLPanalysis [13] and ligation based typing [4].

We have previously developed a homogeneous assay approach for genotyping utilizing asymmetric PCR and lanthanide labelled DNA-probes with LNA bases [8, 9]. Homogeneous assays are separation-free methods were the unreacted label need not be removed from the reaction before measuring the results. The method has been adapted to both extracted DNA samples as well as whole blood samples dried on sample collection cards, the latter being more convenient with the exclusion of a tedious DNA-extraction step [7]. The benefits of this homogeneous approach include reductions in hands-on time as well as whole assay time, reduced contamination risk and, as a concerted consequence of reduced time and material, reduced costs.

The aim of the study was to apply this homogeneous assay principle to an assay for testing the presence of HLA-B*27. We compared the method with an existing HLA-B*27 assay based on DELFIA technology (PerkinElmer Life and Analytical Sciences, Wallac) which we have developed and earlier found to perform 
Table 1

Oligonucleotides used in the assay

\begin{tabular}{|c|c|c|}
\hline Oligonucleotide & Sequence from 5' to 3' end & Label/position \\
\hline $\mathrm{B} * 275^{\prime}$ primer & GTGGGCTACGTGGACGACACGCT & \\
\hline $\mathrm{B} * 27$ 3' primer & CAGTCTGTGCCTTGGCGTTGC & \\
\hline$\beta-\operatorname{actin} 5$ ' primer & TGAAGTGTGACGTGGACATC & \\
\hline$\beta-\operatorname{actin} 3$ ' primer & CTTGATCTTCATTGTGCTGGG & \\
\hline B*27-probe & CGCCGTGGAT & $\mathrm{Tb} / 5$ \\
\hline $\mathrm{B} * 27$ quencer & $\overline{\mathrm{ATCCACG}} \overline{\mathrm{AC}}$ & dabcyl/3' \\
\hline$\beta-$ actin probe & A $\underline{G G G T \underline{A C A T G}}$ & $\mathrm{Eu} / 5$ \\
\hline$\beta-$ actin quencher & CATGTĀCCCT & dabcyl/3' \\
\hline DELFIA-B $* 27$ 5' primer & GCTACGTGGACGACACGCT & \\
\hline DELFIA-B*27 3' primer & CAGTCTGTGCCTTGGCGTTGC & biotin/5 \\
\hline DELFIA- $\beta$-actin $5^{\prime}$, primer & TGAAGTCTGACGTGGACATC & biotin/5 \\
\hline DELFIA- $\beta$-actin 3' primer & CTTGATCTTCATTGTGCTGGG & \\
\hline DELFIA-B*27-probe & GCCGTGGATAGAGC & $20 * \mathrm{Eu} / 5$ \\
\hline DELFIA- $\beta$-actin-probe & AATGCCAGGGTACATG & $20 * \mathrm{Sm} / 5$ \\
\hline
\end{tabular}

favourably compared to traditional methods. Also the suitability of the homogeneous HLA-B*27 assay for a dry reagent approach, consisting of drying all the reagents excluding the sample into the reaction vessel, was tested in order to further increase the simplicity of the assay performance since only the sample with water would then need to be added when performing the assay.

\section{Materials and methods}

\subsection{Oligonucleotides}

The oligonucleotides used in this study and described in Table 1 were purchased from ThermoElectron GmbH (Ulm, Germany) except the probes used in the DELFIA-assay which were purchased from PerkinElmer Life and Analytical Sciences Wallac (Turku, Finland). The other lanthanide probes were labelled at Abacus Diagnostica Ltd. (Turku, Finland).

\subsection{DNA samples}

The EDTA-treated blood was applied to FTA ${ }^{\circledR}$ Classic Card (Whatman International Ltd., Maidstone, UK) sample collection cards and allowed to dry at room temperature. $3 \mathrm{~mm}$ discs were cut from the dried cards into the wells of a ThermoFast ${ }^{\circledR} 96$ PCR plate (ABgene Limited, Epsom, UK). $50 \mu \mathrm{l}$ of $\mathrm{H}_{2} \mathrm{O}$ was added into each well and the plate sealed with a Microseal 'A' Sealing film (Bio-Rad Laboratories, Hercules, CA, USA). The plate was incubated at $100^{\circ} \mathrm{C}$ for ten minutes, cooled to $10^{\circ} \mathrm{C}$ and centrifuged briefly to collect all the liquid to the bottom of the wells. $1 \mu$ l samples of the liquid phase containing DNA liberated from the blood discs were used as template in the PCRs. The concentration of the released DNA was not measured as the assay was optimized to the amount available from blood spots and no indication was found that the concentration would be a critical factor.

\subsection{The homogeneous $H L A-B * 27$-assay}

The PCR components except the DNA polymerase were dried in an Eppendorf tube in vacuum concentrator (Heto-Holten, Denmark). $2 \mathrm{U}$ of DyNAzyme II DNA polymerase (Finnzymes, Espoo Finland) were air-dried into each well of a ThermoFast ${ }^{\circledR} 96$ - PCRplate (skirted, low profile, black), except for the amplification background wells which did not contain the enzyme. The PCR mix was reconstituted with water and $25 \mu \mathrm{l}$ of the reconstituted mix per well was added onto the PCR plate containing the dried polymerase. The reconstituted reactions contained $4.0 \mathrm{mM} \mathrm{MgCl}_{2}, 1 \mathrm{x}$ DyNAzyme $^{\mathrm{TM}}$ reaction buffer, $0.2 \mathrm{mM}$ dNTPs (Fermentas Inc., Helsingborg, Sweden), $0.4 \mu \mathrm{M} \beta$-actin 5'-primer, $0.08 \mu \mathrm{M} \beta$-actin 3'-primer, 0.1 $\mu \mathrm{M}$ HLA$\mathrm{B} * 27$-specific 5'-primer, $0.2 \mu \mathrm{M}$ HLA-B*27-specific 3'-primer and $3.0 \mu \mathrm{g} / \mu \mathrm{l} \mathrm{BSA} \mathrm{(Sigma-Aldrich} \mathrm{Fin-}$ land Oy, Helsinki, Finland). The concentrations of the probe-quencher-pairs were for the $\beta$-actin $3.3 \mathrm{nM}$ (probe) and $6.6 \mathrm{nM}$ (quencher) and for the HLA-B*27 $0.33 \mathrm{nM}$ (probe) and $5.0 \mathrm{nM}$ (quencher).

After $1 \mu \mathrm{l}$ samples of the DNA liberated from the dried blood spots were added, the plates were sealed with Microseal 'A' film and a PCR program was run in a DNA Engine Dyad Peltier Thermal Cycler (Bio-Rad Laboratories) consisting of an initial cycle of $4 \mathrm{~min}$ at $95^{\circ} \mathrm{C}, 2 \mathrm{~min}$ at $64^{\circ} \mathrm{C}$, and $2 \mathrm{~min}$ at $73^{\circ} \mathrm{C}$. This was 
followed by 49 cycles of $30 \mathrm{~s}$ at $95^{\circ} \mathrm{C}, 40 \mathrm{~s}$ at $64^{\circ} \mathrm{C}$, ramping of $0.5^{\circ} \mathrm{C} / \mathrm{s}$ to $73^{\circ} \mathrm{C}$, and $40 \mathrm{~s}$ at $73^{\circ} \mathrm{C}$. After a final extension step of $4 \mathrm{~min}$ at $73^{\circ} \mathrm{C}$, the reactions were slowly cooled to room temperature at an approximate rate of $0.02{ }^{\circ} \mathrm{C} / \mathrm{s}$. All reactions for altogether 229 samples were done in duplicate.

After thermal cycling and cooling, europium and terbium signals were measured at room temperature with a Victor 1420 Multilabel counter (PerkinElmer Life and Analytical Sciences Wallac). Default parameters of the instrument were used for both europium and terbium measurements (for europium excitation and emission wavelengths $340 \mathrm{~nm}$ and $615 \mathrm{~nm}$, respectively, delay $0.4 \mathrm{~ms}$, window time $0.4 \mathrm{~ms}$, and measurement cycle time $1 \mathrm{~ms}$; and for terbium excitation and emission wavelengths $340 \mathrm{~nm}$ and $545 \mathrm{~nm}$, respectively, delay $0.5 \mathrm{~ms}$, window time $1.4 \mathrm{~ms}$, and measurement cycle time $2 \mathrm{~ms}$ ).

To analyze the results, signal-to-background ratios were calculated for both probes by dividing the signal measured from the sample by the signal obtained from a background reaction. For the amplification control the background signal was obtained from a reaction that did not contain the enzyme; and for the HLA-B*27probe a reaction with a negative control sample was used as background. To eliminate variations caused by differences in amplification efficiency, a relative HLA$\mathrm{B} * 27$ ratio was calculated for each sample by dividing the signal-to-background ratio of the HLA-B*27-probe by that of the amplification control probe.

\subsection{The DELFIA HLA-B*27-assay}

The 229 samples were also genotyped with the DELFIA-based method published previously [20]. The DELFIA method consists of a PCR-step used to amplify allele-specifically the HLA-B*27 if present in sample and a section of the $\beta$-actin gene as an amplification control, followed by a hybridisation step where the PCR-products are collected onto a microtitration well and lanthanide labeled probes are used to detect these PCR-products. In short, $25 \mu$ l PCR-reactions consisted of $4 \mathrm{mM} \mathrm{MgCl}_{2}, 0.8 \mathrm{x}$ ABgene PCR-buffer IV (ABgene), $0.4 \mu \mathrm{M} \beta$-actin control primers, $0.1 \mu \mathrm{M}$ HLA$\mathrm{B} * 27$-specific primers, $1.5 \mathrm{M}$ betaine (Sigma-Aldrich Finland Oy), $0.2 \mathrm{mM}$ dNTP's and $0.06 \mathrm{U} / \mu \mathrm{l}$ of ABgene Taq-DNA polymerase. $1 \mu \mathrm{l}$ samples of the extract from the blood spots were added as template per reaction.

The same thermal cycling protocol was used as for the homogeneous assay except for the annealing temperature which was $62^{\circ} \mathrm{C}$ instead of $64^{\circ} \mathrm{C}$ and after the final extension step the reactions were rapidly cooled to $10^{\circ} \mathrm{C}$ omitting the slow cooling.

After the amplification step the DELFIA plate hybridization was performed. The biotinylated PCRproducts were collected to a streptavidin-coated microtiter plate (Kaivogen Oy, Turku, Finland) and rendered single-stranded by denaturing with sodium hydroxide solution and removing the complementary strand by washing. A probe labeled with europium recognizing the HLA-B*27 PCR-product and a probe detecting the $\beta$-actin product labeled with samarium were added and allowed to hybridize to their target sequences and unbound label was removed by washes. The signals were developed and measured and the results of the DELFIA method were calculated as described for the homogeneous assay.

\subsection{The stability of the dried reagents}

The stability of the dried reagents was tested with 0.2 M trehalose (Sigma-Aldrich Finland Oy) as a preserving reagent at room temperature for up to six weeks of storage. The reagents consisting of identical components as described in the homogeneous HLA-B*27 assay with the inclusion of $0.2 \mathrm{M}$ trehalose but without BSA were air-dried onto Thermo-Tube strips (AB0264, ABgene). The DNA-polymerase was air-dried on separate Thermo-Tube strips and sealed into the storage packages together with the reagent strips.

The possibility of including the DNA polymerase in the dried reagent mix was also tested by air-drying the enzyme together with all the other PCR components. All the PCRs were otherwise done as described earlier for the homogeneous HLA-B*27 assay.

\section{Results}

\subsection{The homogeneous HLA-B*27-assay compared to the DELFIA HLA-B*27-assay as a reference method}

Altogether 229 consecutive patient samples tested for the presence of HLA-B*27 using DELFIA method were used to evaluate the performance of the homogeneous assay. Samples were tested in 6 different runs of DELFIA assay with an initial success rate of $84 \%$ and the 36 samples that failed to amplify in the first attempt were successfully typed later. The results of the homogeneous assay are shown in Fig. 1. The samples were analyzed in 5 separate runs with an initial success 


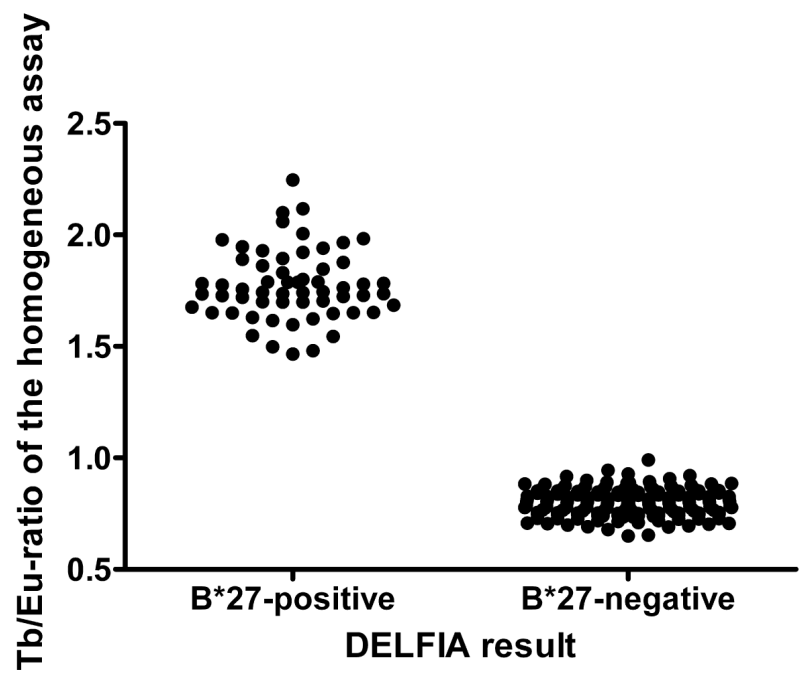

Fig. 1. Results of the HLA-B*27 assay. The relative signal-to-background ratios of 229 samples from 5 separate runs.

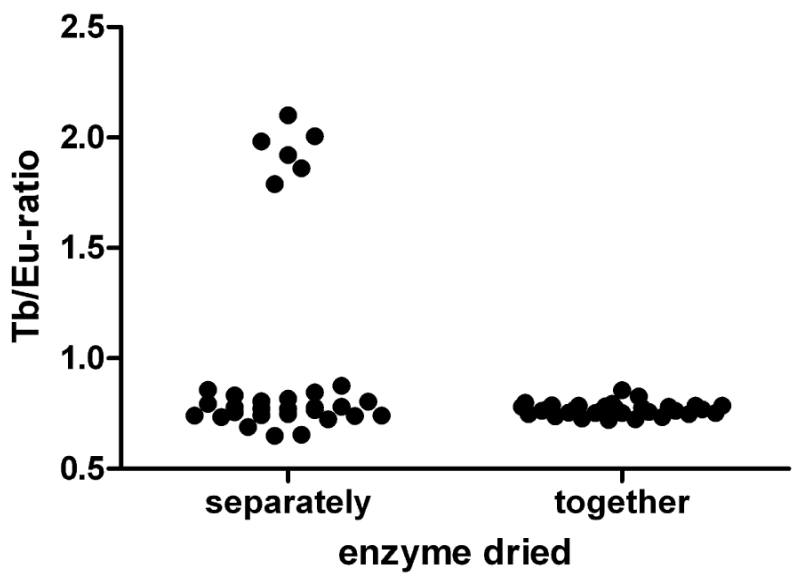

Fig. 2. Testing the possibility of including the DNA-polymerase in the reagent mix. The DNA-polymerase was dried either separately from the other PCR components or included in the PCR mix. As shown by the lack of positive samples in the "together" column the polymerase must be kept separate from the other PCR components.

rate of $96 \%$ and the relative signal ratios calculated as described in Material and Methods for all samples are shown in Fig. 1. The nine samples that failed to reach the limit set for acceptable amplification in the first attempt (not included in the figure) were reanalysed successfully.

All samples were successfully genotyped and also were $100 \%$ concordant when the results of both assay methods were compared.

\subsection{The stability of the dried reagents}

The possibility of including the polymerase in the PCR reagent mix was explored and the results are shown in Fig. 2. The relative HLA-B*27-ratios of those reactions where the enzyme was dried separately are clearly divided into HLA-B*27-positive and - negative samples but when the enzyme was dried together with the rest of the PCR components the positive and negative samples can not be distinguished.

The stability of the dried reagents was initially studied using BSA as a stabilizing agent; but BSA did not prove to be effective enough preserving agent when stored at room temperature, at $+4{ }^{\circ} \mathrm{C}$ or at $-20^{\circ} \mathrm{C}$ for several weeks (results not shown). Trehalose was subsequently tested to be used instead of BSA.

The dry PCR reagents with trehalose were stored at room temperature up to eight weeks. The results of the 


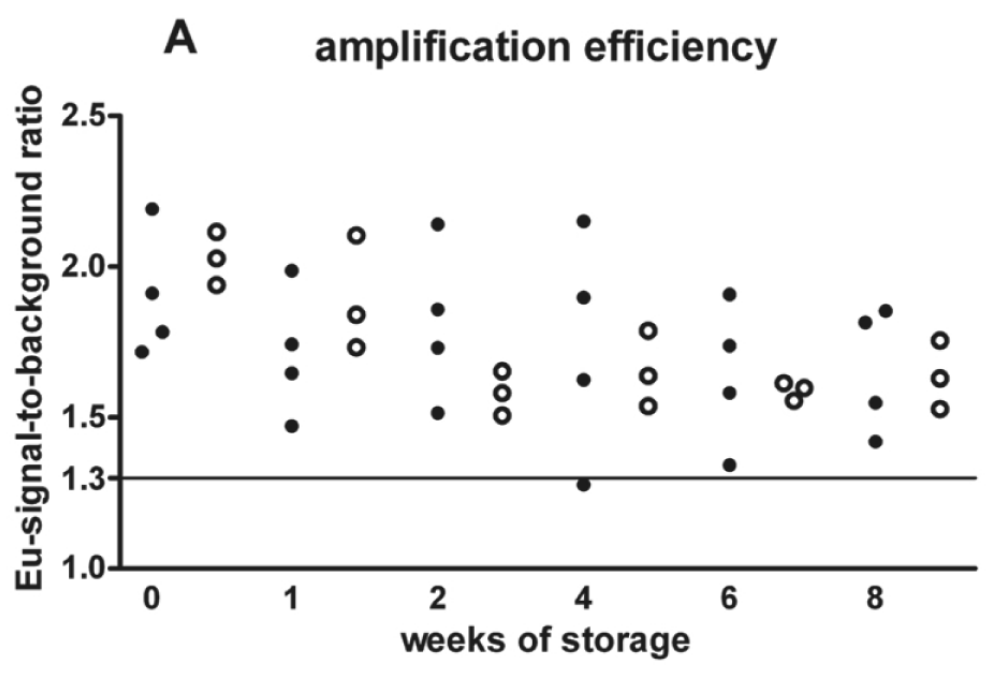

B HLA-B*27-probe

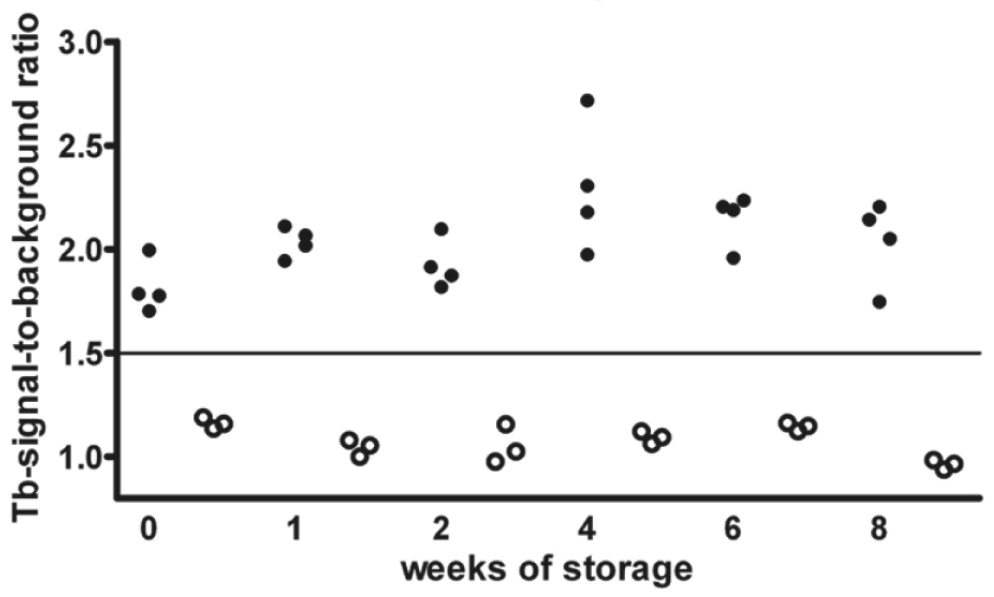

Fig. 3. The results of the dry chemistry reagent stability tests. Panel A shows the signal-to-background ratio of the europium-labeled amplification control probe and panel B the signal-to-background ratio of the HLA-B*27 probe labeled with terbium at six time points from one day to eight weeks of storage. The cut-off values for the amplification control probe and for the HLA-B*27-probe were set at 1.3 and at 1.5 , respectively and are marked with solid horizontal lines in the figure. The B*27-positive samples are marked with filled circles and the B*27-negative samples with open circles.

stability test are shown in Fig. 3 with four HLA-B*27positive and three HLA-B*27-negative samples included in each time point. All samples were successfully typed at each time point with clearly differing positive and negative HLA-B*27-signals as shown by the panel $B$ in Fig. 3. Although at four weeks one HLA-B*27positive sample was slightly below the limit set for the control probe signal-to-background ratio as acceptable amplification as shown in panel A in Fig. 3, but despite this it showed an unambiguously positive HLA-B*27 result. The signal-to-noise ratios of both probes do not show any clear declining trend during the eights weeks and even the raw signals obtained from the reactions (data not shown) remain essentially on the same level at all time points.

\section{Discussion}

We present here a homogeneous method developed for the screening of blood samples for the presence of HLA-B*27 that is simple, robust and fast and also suited for large-scale screening and compares favorably with the previously used DELFIA based assay. In addition the homogeneous method was proven to be adaptable to a dry reagent approach which would fur- 
ther simplify the assay and reduce required hands-on time.

The primers used in the assay have been previously published [14] and are used also in the DELFIA assay [20]. The primers amplify all HLA-B*27-alleles shown to be associated with AS; only few rare alleles are not amplified. A minor modification was done to the HLA-B*27-specific 5' -primer by adding four bases to the 5'-end of the primer to compensate for the diminished concentration of this primer used in the asymmetric PCR when compared to the HLA-B*27-specific 3'-primer concentration, since the $\mathrm{T}_{m}$ of the primers depends on the concentration as well as the length of the primer $[8,15]$. The inclusion of LNA-bases in the lanthanide-labeled probes was shown by our previous work to improve significantly the performance of the probes, when compared to regular, only-DNA containing oligonucleotides, in the developed assay format [8] and although the specificity in this assay relies mainly on the HLA-B*27-primers, given that the HLA-B*27 probe used in the homogeneous assay has a sequence which can be found also in other HLA-B-alleles, such as HLA-B*07, *08 and *39 among others, we chose to take advantage of the strong affinity of LNA-bases [2, 12] in this assay as well to allow the use of short probes.

The developed method was optimized to ensure the slightly preferential amplification of the HLA-B*27 over the $\beta$-actin amplification control to guarantee that no false negative results would be produced. In some cases even though the signal-to-background ratios of the control probe did not reach the set cut-off value, indicating insufficient amplification, the HLA-B*27probe showed a clear positive result (data not shown), indicating that the chosen conditions truly favor the amplification of the HLA-B*27 over the amplification control.

Compared with the DELFIA method the interassay variation was smaller with the homogeneous assay enabling easy comparison of separate assay runs, although all the results were also easily interpreted within each of the DELFIA assays. Also the initial success rate of the developed homogeneous method is superior to that of the DELFIA method demonstrating a more robust approach.

A dry reagent approach would simplify even further the developed method since only the pipetting of the samples and possibly adding of water would be required. With this in mind the stability of the PCR components in dry format was tested with an air-drying process that requires no specialized equipment. The time required for preparing the dry reagents consists of ap- proximately 30 minutes of hands-on time for preparing the reagents and dispensing them into the reaction vessels and of overnight drying after which the reagents are ready to use or can be packaged for storage. With BSA as a stabilizing agent the stability of the dried reagents was not sufficient and therefore we tested trehalose that has been shown to protect dried reagents [10] as well as act as a PCR enhancer $[16,18]$. The reagents air-dried together with $0.2 \mathrm{M}$ trehalose with separately dried DNA-polymerase were shown to be stable at least up to eight weeks of storage at room temperature. Since the signal-to-noise ratios of either the HLA-B*27-probe or the amplification control probe did not show any clear decline during this period it could be assumed that even longer storage time could be used, and these tests are continuing in our laboratory. Storing the reagents in lower temperatures, such as at $+4^{\circ} \mathrm{C}$ or even at $-20^{\circ} \mathrm{C}$, would likely result in even longer self-life than expected for storage at room temperature [5,6].

When the possibility of including the polymerase with the other reaction components was tested, the results showed clearly that the enzyme could not be dried together with the other PCR components. However, the enzyme might be dried in the same reaction vessel with the other components provided that the enzyme remains physically separate from the other components during all of the time of storage [19]. For example, the enzyme could be dried to a different position in the PCR vessel while the rest of the mix was on the bottom, but this would require more sophisticated instrumentation than were available for this study. When this is accomplished only the sample needs to be added to the reaction well in order to perform the homogenous amplification assay and the assay can be run in a few hours or less depending on the speed of the thermal cycler available.

The homogeneous approach also proved to be somewhat cheaper than the DELFIA format when the reagent and material costs were calculated. Much of the diminished costs of the homogeneous assay are due to the absent hybridization step since the probes used to recognize the PCR-products are already included in the PCR mix and this is true also when compared to other methods used to type for the presence of HLA$\mathrm{B} * 27$ requiring a separate detection step, such as plate hybridization or gel electrophoresis. Also the loss of reagents is smaller if pre-dried reagents are used, since usually the mixtures used in PCR and hybridization are prepared with some excess to facilitate pipetting. More importantly, the time saved in hands-on time with the homogeneous method reduces the costs of the assay 
even more significantly, since labor is in many occasions the most expensive element. Since the separate hybridization step, requiring the greatest part of the hands-on work for example in the DELFIA method, is omitted from the homogeneous method the whole assay can be run in approximately 4 hours with 1 hour or less of hands-on time compared to the minimum of 8 hours required by the DELFIA method with more than 3 hours of hands-on time.

The flexibility of the developed homogeneous HLA$\mathrm{B} * 27$ assay allows either the typing of only a few samples at a time or it can be applied to the screening of hundreds of samples, according to needs of the user, without any modifications to the assay protocol. With the minimized assay and hands-on time, especially when using the pre-dried reagents, and the reduced total costs, the presented method compares favorably with the existing assays in the typing of samples for the presence of HLA-B*27.

\section{References}

[1] P. Bowness, HLA B27 in health and disease: a double-edged sword, Rheumatology 41 (2002), 857-868.

[2] D.A. Braasch and D.R. Corey, Locked nucleic acid (LNA): fine-tuning the recognition of DNA and RNA, Chem Biol 8 (2001), 1-7.

[3] D.A. Brewerton, F.D. Hart, A. Nicholls, M. Caffrey, D.C. James and R.D. Sturrock, Ankylosing spondylitis and HL-A 27, Lancet 1 (1973), 904-907.

[4] G.F. Fischer, I. Fae, S. Moser, M. Petrasek, H.M. Bassler, G.R. Menzel and W.R. Mayr, Ligation based HLA-B*27 typing, Tissue Antigens 48 (1996), 148-152.

[5] K. Glynou, P. Kastanis, S. Boukouvala, V. Tsaoussis, P.C. Ioannou, T.K. Christopoulos, J. Traeger-Synodinos and E. Kanavakis, High-throughput microtiter well-based chemiluminometric genotyping of $15 \mathrm{HBB}$ gene mutations in a dryreagent format, Clin Chem $\mathbf{5 3}$ (2007), 384-391.

[6] V. Hagren, P. von Lode, A. Syrjälä, T. Soukka, T. Lövgren, H. Kojola and J. Nurmi, An automated PCR platform with homogeneous time-resolved fluorescence detection and dry chemistry assay kits, Anal Biochem 374 (2008), 411-416.

[7] M. Kiviniemi, R. Hermann, J. Nurmi, A.G. Ziegler, M. Knip, O. Simell, R. Veijola, T. Lövgren, J. Ilonen and the TEDDY
Study Group, A high-throughput population screening system for the estimation of genetic risk for type 1 diabetes: an application for the TEDDY (the Environmental Determinants of Diabetes in the Young) study, Diabetes Technol Ther 9 (2007), 460-472.

[8] M. Kiviniemi, J. Nurmi, T. Lövgren and J. Ilonen, Locked nucleic acid (LNA) probes in high-throughput genetic analysis: Application to an assay for type 1 diabetes-related HLADQB1 alleles, Clin Biochem 38 (2005), 1015-1022.

[9] M. Kiviniemi, J. Nurmi, H. Turpeinen, T. Lövgren and J. Ilonen, A homogeneous high-throughput genotyping method based on competitive hybridization, Clin Biochem 36 (2003), 633-640.

[10] P.R. Klatser, S. Kuijper, C.W. van Ingen and A.H.J. Kolk, Stabilized, Freeze-Dried PCR Mix for Detection of Mycobacteria, J Clin Microbiol 36 (1998), 1798-1800.

[11] J. Klein and A. Sato, The HLA system. Second of two parts, N Engl J Med 343 (2000), 782-786.

[12] A.A. Koshkin, P. Nielsen, M. Melgaard, V.K. Rajwanshi, S.K. Singh and J. Wengel, LNA (locked nucleic acid): An RNA mimic forming exceedingly stable LNA:LNA duplexes, $J$ Am Chem Soc 120 (1998), 13252-13253.

[13] A. Nieto, A. Fraile, J. Vinasco and J. Martín, HLA-B*27 typing by PCR - restriction fragment length polymorphism, Tissue Antigens 49 (1997), 283-286.

[14] O. Olerup, HLA-B27 typing by a group-specific PCR amplification, Tissue Antigens 43 (1994), 253-256.

[15] J.A. Sanchez, K.E. Pierce, J.E. Rice and L.J. Wangh, LinearAfter-The-Exponential (LATE)-PCR: An advanced method of asymmetric PCR and its uses in quantitative real-time analysis, Proc Natl Acad Sci USA 101 (2004), 1933-1938.

[16] A.N. Spiess, N. Mueller and R. Ivell, Trehalose Is a Potent PCR Enhancer: Lowering of DNA Melting Temperature and Thermal Stabilization of Taq Polymerase by the Disaccharide Trehalose, Clin Chem 50 (2004), 1256-1259.

[17] E. Thorsby and B.A. Lie, HLA associated genetic predisposition to autoimmune diseases: Genes involved and possible mechanisms, Transplant Immunology 14 (2005), 175-182.

[18] J.A. Tomlinson, N. Boonham, K.J. Hughes, R.L. Griffin and I. Barker, On-site DNA extraction and real-time PCR for detection of Phytophthora ramorum in the field, Appl Environ Microbiol 71 (2005), 6702-6710.

[19] P. von Lode, A. Syrjälä, V. Hagren, H. Kojola, T. Soukka, T. Lövgren and J. Nurmi, Fully Automated, Homogeneous Nucleic Acid Detection Technology Based on Dry-Reagent Assay Chemistry and Time-Resolved Fluorometry, Clin Chem 53 (2007), 2014-2017.

[20] L. Välimaa, M. Sjöroos, M. Luhtala, P. Toivanen, T. Lövgren and J. Ilonen, Detection of HLA-B27 alleles by group-specific amplification and time - resolved fluorometry, J Immunol Methods 219 (1998), 131-137. 


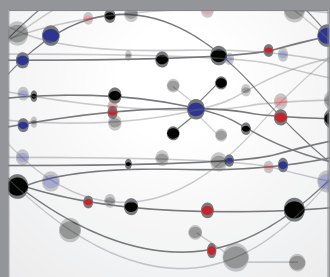

The Scientific World Journal
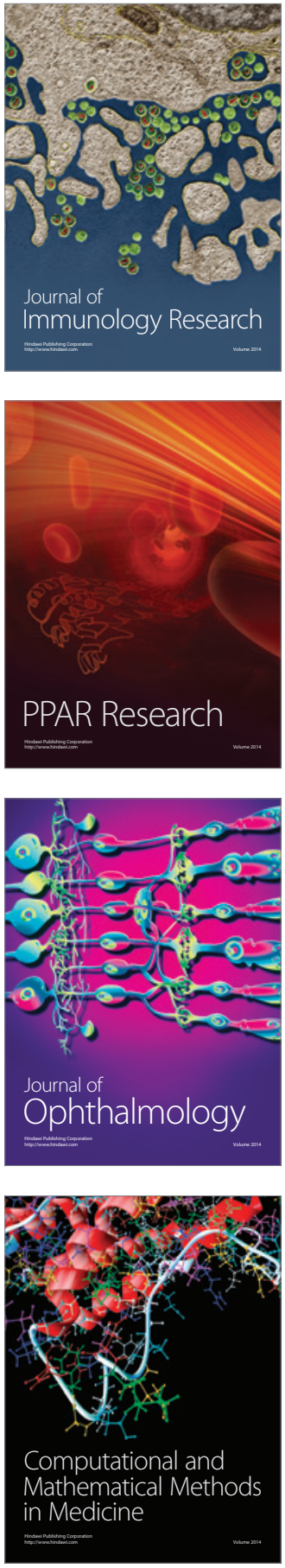

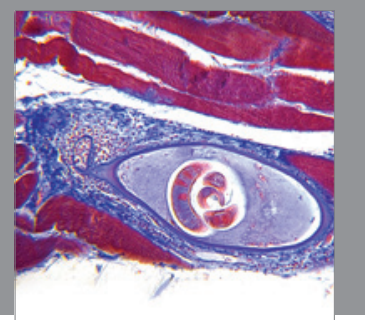

Gastroenterology

Research and Practice
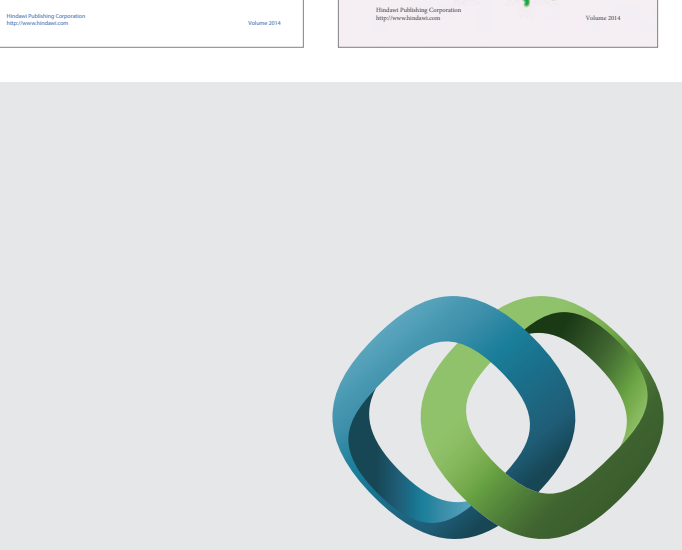

\section{Hindawi}

Submit your manuscripts at

http://www.hindawi.com
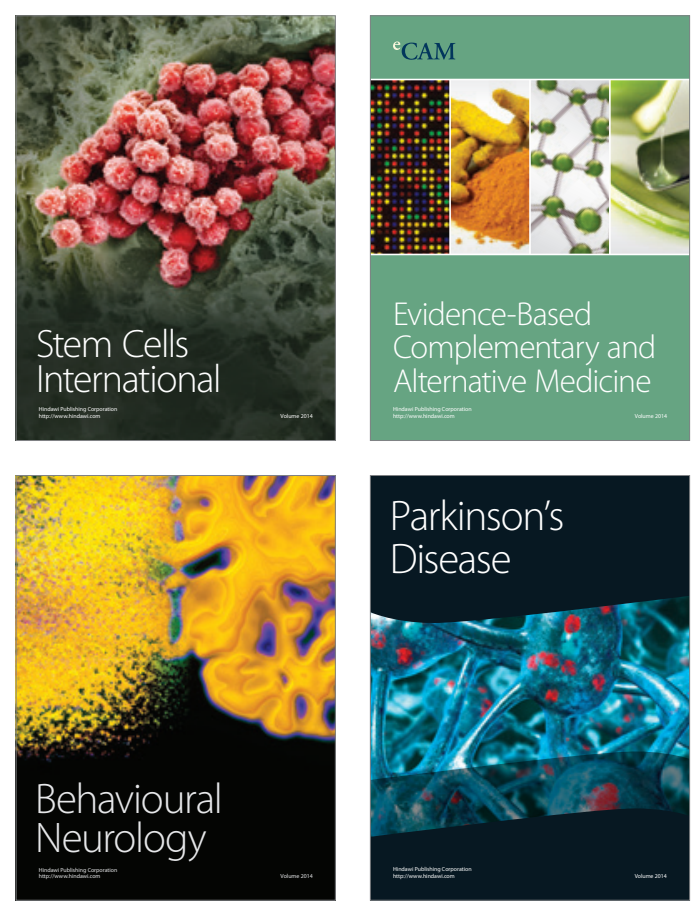

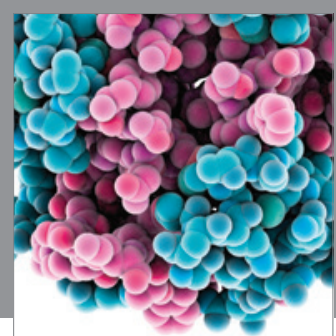

Journal of
Diabetes Research

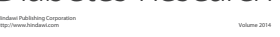

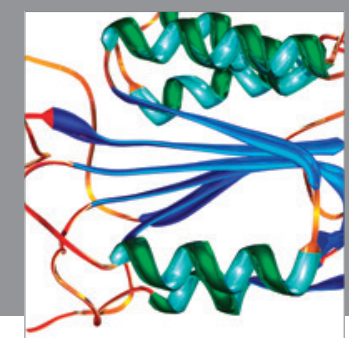

Disease Markers
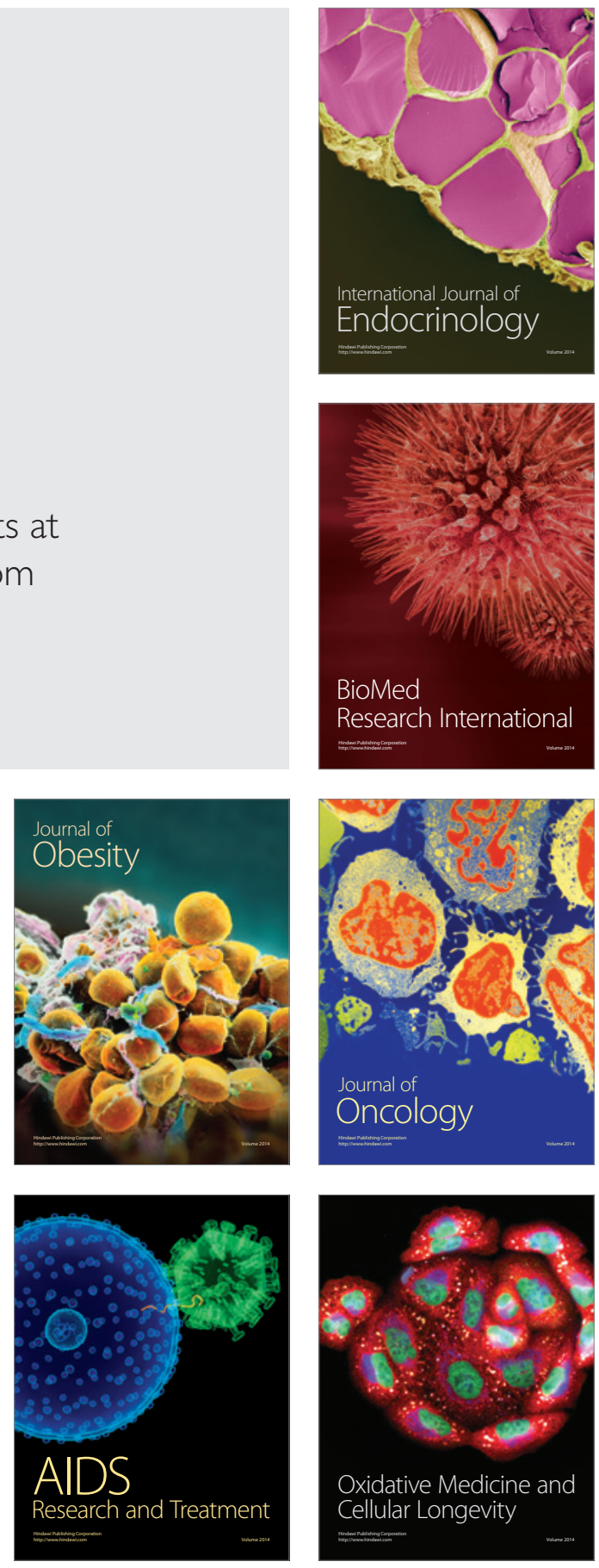